\title{
Mass Estimation of White Dwarfs in Intermediate Polars using the Suzaku Satellite
}

\author{
Takayuki Yuasa*, Kazuhiro Nakazawa, \\ Department of Physics, School of Science, The University of Tokyo, Japan \\ E-mail: yuasa@juno.phys.s.u-tokyo.ac.jp \\ Kauzo Makishima, \\ Department of Physics, School of Science, The University of Tokyo, Japan and RIKEN, Japan
}

\section{Kei Saitou, Ken Ebisawa, and}

ISAS/JAXA, Japan and Department of Astronomy, School of Science, The University of Tokyo, Japan

\section{Manabu Ishida \\ ISAS/JAXA, Japan}

\begin{abstract}
Using wide-band X-ray spectra of nearby Intermediate Polars (IPs) measured with Suzaku, we estimated white dwarf masses in each system, and compared them with that of non-magnetic cataclysmic variables. In addition to classical IPs whose masses are in good agreement with previous reports, we also successfully estimated masses of two IPs recently found by the INTEGRAL mission.
\end{abstract}

The Extreme sky: Sampling the Universe above $10 \mathrm{keV}$ - extremesky2009,

October 13-17, 2009

Otranto (Lecce) Italy

\footnotetext{
* Speaker.
} 


\section{Introduction}

An Intermediate Polar (IP; e.g. Patterson 1994) is a binary system which consists of a moderately magnetized white dwarf (WD; $B \sim 0.1-10 \mathrm{MG}$ ) and a Roche-lobe-filling low mass companion, and is a subclass of magnetic cataclysmic variables. Because of its intense three Fe lines and high temperature optically thin thermal continuum $(k T>10 \mathrm{keV})$, some authors have been suggesting that they are the origin of the apparently extended X-ray emission along the Galactic plane (called Galactic ridge emission; Revnivtsev et al. 2006; Krivonos et al. 2007 and references therein) especially in the hard X-ray band.

To study this idea by comparing their spectra in the hard X-ray band where an IP thermal spectrum is thought to have an exponential cut off, we have been observing IPs using Suzaku. In this paper, we estimate WD masses of 17 IPs by fitting spectra with an emission model which involves few physical assumptions, and obtain their average mass to enable future comparison between IP spectra and that of the Galactic ridge emission.

\section{Methods}

\subsection{How to estimate WD masses?}

In magnetic CVs, gas accreting from a companion low-mass star is channeled along a strong magnetic field of a WD, and falls onto magnetic poles of the WD with almost its free-fall velocity. Near the WD surface, the velocity exceeds the sound velocity of the gas, and a shock stands thereby. The shock converts the energy of the bulk motion of the gas into the internal energy, in other words, the gas is heated. The temperature of the shock-heated gas simply depends on the depth of the gravitational potential of the WD. Therefore, by equating a free-fall velocity and a boundary condition of a strong shock, the temperature can be written as,

$$
k T=\frac{3}{8} \frac{G M}{R} \mu m_{\mathrm{H}}=16\left(\frac{M_{\mathrm{WD}}}{0.5 M_{\odot}}\right)\left(\frac{R_{\mathrm{WD}}}{10^{9} \mathrm{~cm}}\right)^{-1} \mathrm{keV} .
$$

With typical parameters of WDs, this temperature exceeds $10 \mathrm{keV}$, and thus a post-shock region filled with the heated gas emits hard X-rays. We can measure this temperature using X-ray data, and that results a relation between $M_{\mathrm{WD}} / R_{\mathrm{WD}}$. Since another WD mass-radius relation has been also constructed theoretically (e.g. Nauenberg 1972), $M_{\mathrm{WD}}$ and $R_{\mathrm{WD}}$ can be separately calculated by equating them. Using many X-ray satellites, multiple authors have been performing this estimation using, mainly, continuum spectra of magnetic CVs (Ishida 1991; Cropper et al. 1999; Ramsay 2000; Suleimanov et al. 2005; Brunschweiger et al. 2009).

Because of the high temperature, it requires a sensitive hard X-ray detector to measure the shock-temperature accurately based on a continuum spectrum. In addition to that, emission lines from heavy atoms also serves information of the gas temperature, and therefore, good energy resolution is appreciated especially below $10 \mathrm{keV}$. From these viewpoints, Suzaku, which has two complementary detectors of X-ray Imaging Spectrometer (XIS; Koyama et al. 2007) and Hard Xray Detector (HXD; Takahashi et al. 2007; Kokubun et al. 2007), is very suitable for this study providing the wide energy coverage. 

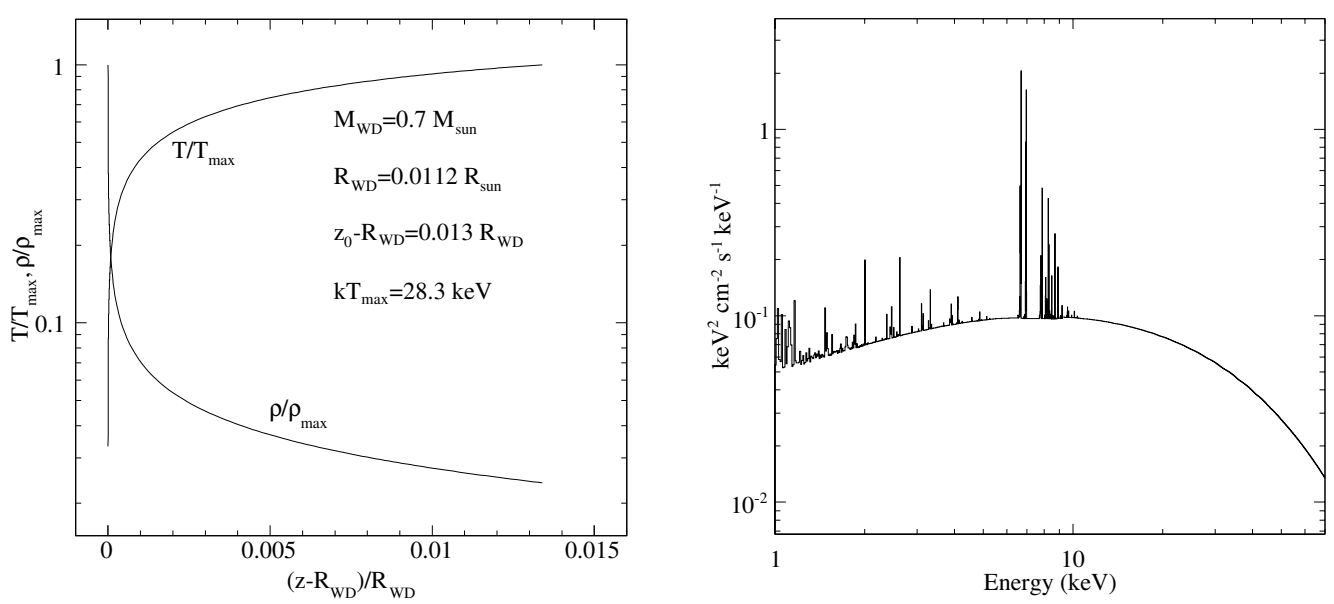

Figure 1: Left: Normalized density and temperature profiles of an accretion column for a $0.7-M_{\odot}$ WD. Abscissa is a height from the WD surface. Right: The expected emission spectrum from a multi-temperature accretion column viewed from an assumed distance of $100 \mathrm{pc}$.

\subsection{Model of an accretion column}

In the present study, we utilized a 1-dimensional accretion column model of Suleimanov et al. (2005) which takes into account the gravitational force of a WD inside the column. By assuming a free-fall ideal gas stream, a strong shock, a optically thin thermal cooling only, and soft landing onto WD atmosphere, we numerically solved the continuity, momentum conservation, energy conservation, and ideal-gas law, and obtained profiles of density and temperature for an assumed $M_{\mathrm{WD}}$ which is a free parameter. We present such profiles calculated for $M_{\mathrm{WD}}=0.7 M_{\odot}$ in Figure 1] To construct a total emission spectrum from the accretion column and to perform spectral fittings, we convolved the profiles with a spectrum of a single temperature plasma emission (apec; Smith et al. 2001).

\section{Result and Brief Disucussion}

We fitted 17 IP spectra obtained with Suzaku XIS/HXD over the 3-40 keV band using the spectral model calculated above. We took into account a patchy absorption (i.e. partial covering) caused by pre-shock gasses as previous reports (e.g. Cropper et al. 1999; Suleimanov et al. 2005). Table 1 lists the obtained best fitting WD masses. As Figure 2 shows examples of EX Hya, IGR J17195 and IGR J17303, the fits were acceptable in all cases. Basically, the resulting WD masses of classical IPs are in good agreement with the previous studies. In addition to that, it should be noted that WD masses of two IGR sources, IGR J17195 and IGR J17303, were also estimated to be $0.91_{-0.12}^{+0.12} M_{\mathrm{WD}}$ and $1.06_{-0.21}^{+0.16} M_{\mathrm{WD}}$.

In Figure 3 we compare the derived $M_{\mathrm{WD}}$ distribution with that of non-magnetic CVs (nmCVs) compiled by Ritter \& Kolb (2003). Mean values are 0.92 and $0.83 M_{\mathrm{WD}}$ for IP and nmCV, respectively. Although our IP sample is still small, the present result does not show large deviation from the nmCVs mass distribution. We expect that the sample will be increased by observing other 
INTEGRAL-found IPs (Barlow et al. 2006) that have no $M_{\mathrm{WD}}$ estimations so far. The distribution of $\mathrm{Fe}$ abundances are also shown in Figure 3. All the values are sub-solar, and this confirms the result of Ezuka \& Ishida (1999) with much smaller fitting errors.
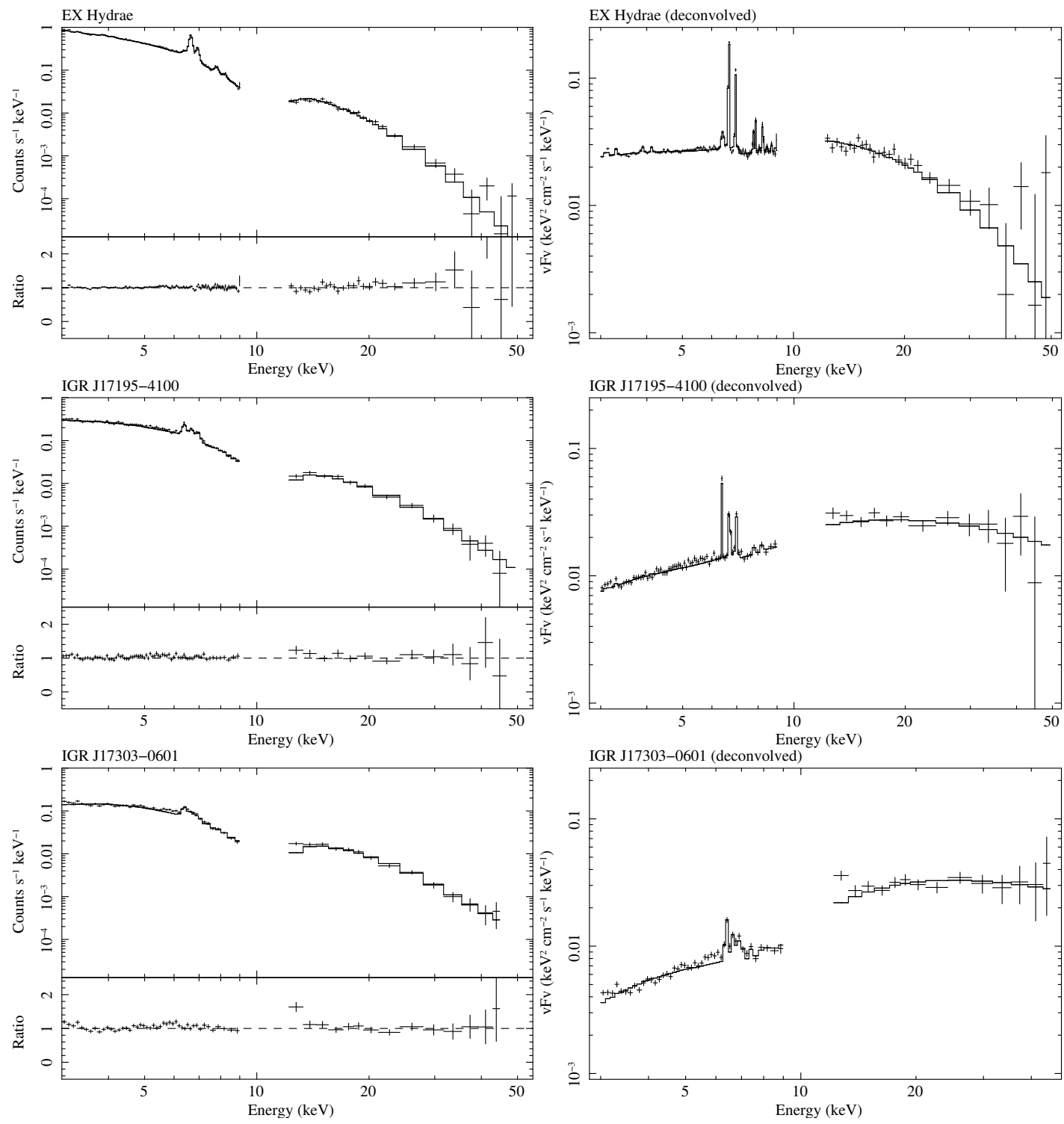

Figure 2: Examples of best-fit spectra for EX Hya, IGR J17195, and IGR J17303. Left and right panels show spectra of individual sources and the best-fit models before and after deconvolving the detector responses.

\section{References}

Barlow, E. J., Knigge, C., Bird, A. J., et al. 2006, MNRAS, 372, 224

Brunschweiger, J., Greiner, J., Ajello, M., \& Osborne, J. 2009, A\&A, 496, 121

Cropper, M., Wu, K., Ramsay, G., \& Kocabiyik, A. 1999, MNRAS, 306, 684 

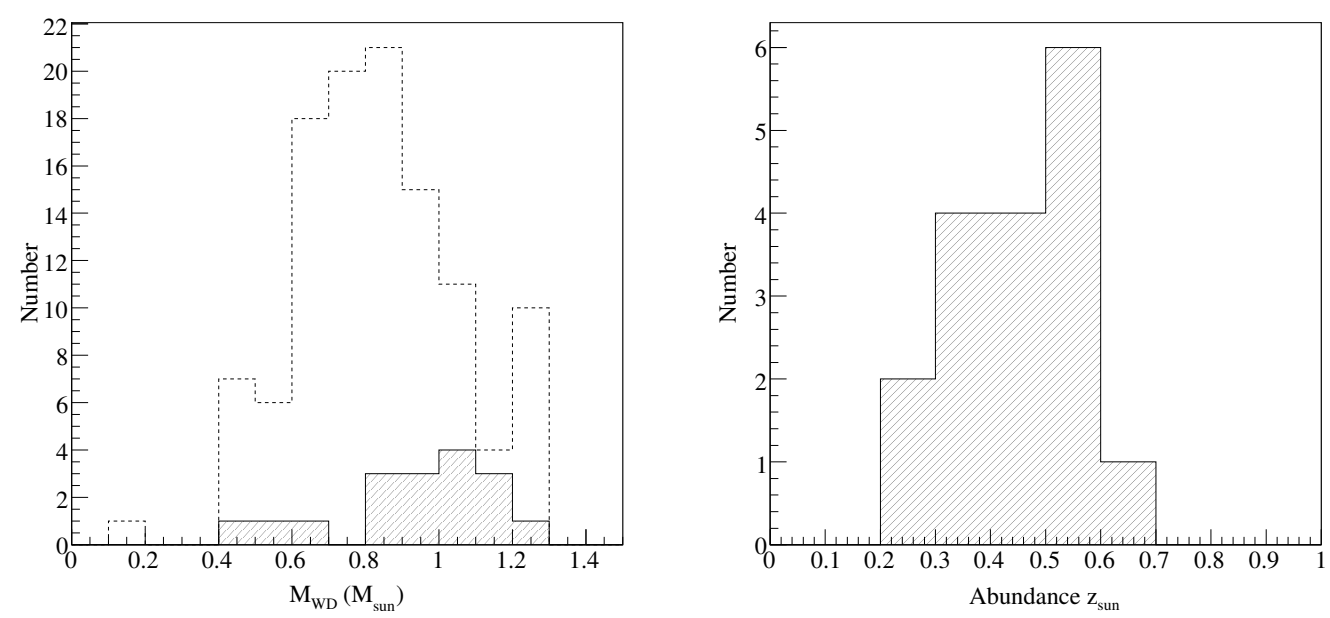

Figure 3: Left: $M_{\mathrm{WD}}$ distribution for 17 IPs (hatched region; this study) and 114 non-magnetic CVs (dashed line; Ritter \& Kolb 2003). Right: A histogram of derived Fe abundances of 17 IPs.

Ezuka, H. \& Ishida, M. 1999, ApJS, 120, 277

Ishida, M. 1991, PhD thesis, The University of Tokyo

Kokubun, M., Makishima, K., Takahashi, T., et al. 2007, PASJ, 59, 53

Koyama, K., Tsunemi, H., Dotani, T., et al. 2007, PASJ, 59, 23

Krivonos, R., Revnivtsev, M., Churazov, E., et al. 2007, A\&A, 463, 957

Nauenberg, M. 1972, ApJ, 175, 417

Patterson, J. 1994, PASP, 106, 209

Ramsay, G. 2000, MNRAS, 314, 403

Revnivtsev, M., Sazonov, S., Gilfanov, M., Churazov, E., \& Sunyaev, R. 2006, A\&A, 452, 169

Ritter, H. \& Kolb, U. 2003, A\&A, 404, 301

Smith, R. K., Brickhouse, N. S., Liedahl, D. A., \& Raymond, J. C. 2001, ApJL, 556, L91

Suleimanov, V., Revnivtsev, M., \& Ritter, H. 2005, A\&A, 435, 191

Takahashi, T., Abe, K., Endo, M., et al. 2007, PASJ, 59, 35 
Table 1: The best-fit spectral parameters obtained with the partial-covering absorber model.

\begin{tabular}{lllllll}
\hline \hline System & $\begin{array}{l}Z_{\mathrm{Fe}}^{\mathrm{a}} \\
\left(Z_{\odot}\right)\end{array}$ & $\begin{array}{l}M_{\mathrm{WD}} \\
\left(M_{\odot}\right)\end{array}$ & $\begin{array}{l}k T_{\mathrm{s}}^{\mathrm{b}} \\
(\mathrm{keV})\end{array}$ & $\chi_{v}^{2}(\mathrm{v})$ & $F_{2-10^{\mathrm{c}}}$ & $F_{12-40}{ }^{\mathrm{d}}$ \\
\hline FO Aqr & $0.4(0.30-0.41)$ & $1.00_{-0.09}^{+0.08}$ & $55.8_{-10.0}^{+11.0}$ & $1.07(1376)$ & 3.82 & 5.44 \\
XY Ari & $0.5(0.45-0.51)$ & $1.04_{-0.09}^{+0.12}$ & $61.0_{-11.0}^{+19.8}$ & $1.03(1496)$ & 1.51 & 2.79 \\
MU Cam & $0.6(0.52-0.71)$ & $0.95_{-0.13}^{+0.04}$ & $49.9_{-12.5}^{+4.6}$ & $1.01(627)$ & 0.90 & 1.83 \\
BG CMi & $0.2(0.19-0.29)$ & $1.15_{-0.17}^{+0.07}$ & $78.8_{-25.4}^{+15.7}$ & $0.99(937)$ & 1.97 & 3.04 \\
V709 Cas & $0.2(0.19-0.26)$ & $1.23_{-0.10}^{+0.05}$ & $97.2_{-22.1}^{+15.9}$ & $0.94(1307)$ & 3.70 & 5.41 \\
TV Col & $0.5(0.48-0.53)$ & $0.94_{-0.10}^{+0.10}$ & $48.9_{-9.7}^{+12.1}$ & $1.03(1390)$ & 4.52 & 5.85 \\
TX Col & $0.5(0.40-0.63)$ & $0.89_{-0.14}^{+0.32}$ & $43.8_{-11.9}^{+48.2}$ & $1.13(721)$ & 1.10 & 1.11 \\
YY Dra & $0.5(0.38-0.64)$ & $0.67_{-0.08}^{+0.05}$ & $26.3_{-4.93}^{+3.41}$ & $1.02(945)$ & 3.35 & 2.80 \\
PQ Gem & $0.3(0.19-0.32)$ & $1.15_{-0.10}^{+0.10}$ & $78.8_{-24.2}^{+24.2}$ & $0.99(1024)$ & 2.18 & 2.81 \\
EX Hya & $0.5(0.49-0.57)$ & $0.39_{-0.01}^{+0.01}$ & $11.4_{-0.42}^{+0.43}$ & $1.05(1666)$ & 8.14 & 3.54 \\
NY Lup & $0.5(0.49-0.55)$ & $1.14_{-0.05}^{+0.06}$ & $76.9_{-8.6}^{+12.6}$ & $1.08(1531)$ & 3.10 & 5.91 \\
V2400 Oph & $0.4(0.39-0.46)$ & $0.77_{-0.05}^{+0.05}$ & $32.3_{-3.8}^{+4.2}$ & $1.08(1635)$ & 4.24 & 6.21 \\
GK Per & $0.3(0.14-0.50)$ & $1.27(>0.72)$ & $110(>30)$ & $0.98(308)$ & 0.76 & 0.61 \\
AO Psc & $0.4(0.38-0.45)$ & $0.54_{-0.03}^{+0.03}$ & $18.6_{-1.6}^{+1.7}$ & $1.08(1398)$ & 4.42 & 4.19 \\
V1223 Sgr & $0.3(0.30-0.35)$ & $0.86_{-0.07}^{+0.05}$ & $41.0_{-6.0}^{+4.8}$ & $1.15(1675)$ & 9.28 & 15.6 \\
RX J2133 & $0.4(0.39-0.43)$ & $1.04_{-0.29}^{+0.06}$ & $61.0_{-29.1}^{+9.0}$ & $1.12(1492)$ & 2.10 & 4.56 \\
IGR J17303 & $0.3(0.27-0.33)$ & $1.06_{-0.16}^{+0.16}$ & $63.8_{-23.7}^{+30.7}$ & $1.06(758)$ & 1.85 & 5.77 \\
IGR J17195 & $0.4(0.38-0.43)$ & $0.91_{-0.12}^{+0.12}$ & $45.7_{-10.8}^{+13.9}$ & $0.88(1190)$ & 3.38 & 4.93 \\
\hline
\end{tabular}

${ }^{\text {a }}$ The abundance of the best-fit model $\left(0.1 Z_{\odot}\right.$ step) followed by the $90 \%$ confidence range.

${ }^{\mathrm{b}}$ The shock temperature corresponding to the WD mass. ${ }^{\mathrm{c}} 2-10 \mathrm{keV}$ flux in units of $10^{-11} \mathrm{erg} \mathrm{cm}^{-2} \mathrm{~s}^{-1} \cdot{ }^{\mathrm{d}} 12-40 \mathrm{keV}$ flux in units of $10^{-11} \mathrm{erg} \mathrm{cm}^{-2} \mathrm{~s}^{-1}$. 